\title{
Übersichten
}

coloproctology $2019 \cdot 41: 272-279$

https://doi.org/10.1007/s00053-019-0362-0

Online publiziert: 7. Mai 2019

(c) Springer Medizin Verlag GmbH, ein Teil von Springer Nature 2019

\author{
A. D. Rink $\cdot$ F. Aigner ${ }^{2} \cdot$ M. Biebl $l^{2} \cdot$ A. Fürst ${ }^{3} \cdot$ W. Kneist ${ }^{4}$ \\ ${ }^{1}$ Klinik für Allgemein-, Viszeral- und Thoraxchirurgie, Klinikum Leverkusen, Leverkusen, Deutschland \\ ${ }^{2}$ Chirurgische Klinik, Campus Charité Mitte, Campus Virchow-Klinikum, Charité - Universitätsmedizin \\ Berlin, Berlin, Deutschland \\ ${ }^{3}$ Klinik für Allgemein-, Viszeral- und Thoraxchirurgie, Caritas Krankenhaus St. Josef, Regensburg, \\ Deutschland \\ ${ }^{4}$ Klinik für Allgemein-, Viszeral- und Transplantationschirurgie, Universitätsmedizin Mainz, Mainz, \\ Deutschland
}

\section{Transanale totale mesorektale Exzision - eine sinnvolle Operationstechnik zur individualisierten Behandlung von Patienten mit Rektumkarzinom}

Die Technik der totalen mesorektalen Exzision (TME) hat zweifelsfrei zu einer erheblichen Verbesserung der Prognose des Rektumkarzinoms geführt. Durch intensive weltweite Schulungen konnten die Lokalrezidivraten auch in populationsbasierten Studien auf unter $10 \%$ reduziert werden. Trotzdem kennt jeder Chirurg die Situationen, bei denen ein optimales Resektionsergebnis mit der konventionellen TME, egal ob in laparoskopischer oder in offener Technik, nur schwer zu erzielen ist.

Die transanale TME (TaTME) ist eine Methode, mit der möglicherweise die Rate an suboptimalen Resektionen verringert werden kann. Hierfür besteht eine gute Rationale. Der vorliegende Beitrag soll den derzeitig Stand bei der Etablierung der TaTME sowie diese Rationale und die Evidenz für eine mögliche Verbesserung der Ergebnisse bei der TME zur Behandlung des Rektumkarzinoms beleuchten.

\section{Die Rationale für die Etablierung neuer Techniken in der Chirurgie des Rektumkarzinoms}

\section{In vielen Studien zur TME finden sich hohe Raten an permanenten Kolostomien}

Die beachtlichen onkologischen Ergebnisse der aktuellen Rektumkarzinomstudien werden immer noch unter Inkaufnahme sehr hoher Raten an permanenten Kolostomien erreicht. Allein präoperativ eingeschränkte Funktion und Patientenwunsch begründen dies sicher nicht In der COLOR-2-Studie lag die Rate an permanenten Kolostomien in der offenen Gruppe bei $30 \%$, in der Gruppe mit laparoskopischer TME (LapTME) sogar bei $35 \%$, und das, obwohl ein Drittel der eingeschlossenen Patienten einen Tumor bei $10-15 \mathrm{~cm}$ hatte, bei dem eine permanente Kolostomie eigentlich eine Ausnahme darstellt [1]. In der amerikanischen ACOSOG-Z6051-Studie lag die Exstirpationsquote mit $21 \%$ nach offener und $24 \%$ nach laparoskopischer Operation ebenfalls hoch [2]. In der australischen ALaCaRT war die Quote mit 7-8\% und in der OCUM-Studie mit 12,8\%
(2,4\% bei Tumoren des mittleren Drittels, aber auch $35,7 \%$ bei Tumoren im unteren Drittel) insgesamt niedriger[3]. Insbesondere die hohe Exstirpationsquote in der COLOR-2-Studie legt nahe, dass in nicht wenigen Fällen eine Exstirpation durchgeführt wurde, weil der Operateur sich alleine auf abdominalem Weg nicht in der Lage sah, eine onkologisch adäquate Resektion zu erreichen. Technische Möglichkeiten, die es erlauben, die gleiche Resektionsqualität zu erreichen, ohne den analen Sphinkterapparat zu opfern, scheinen daher wünschenswert.

\section{Die transabdominale TME führt nicht selten zu suboptimalen Präparaten}

In der Aufarbeitung der pathologischen Präparate der holländischen Multicenterstudie zur neoadjuvanten Kurzzeittherapie beim Rektumkarzinom fanden sich bei $61 \%$ der Patienten mit distalen Rektumkarzinomen suboptimale TME-Präparate, und auch für Tumoren mit Unterrand bei $5-10 \mathrm{~cm}$ lag die Quote bei 35\% [4]. Auch in den aktuelleren größeren Studien werden in 8-29\% der Fälle keine optimalen TME-Präparate erreicht. In 


\begin{tabular}{|c|c|c|c|c|c|c|c|c|}
\hline \multirow[b]{3}{*}{ De'Angelis 2015 [15] } & \multirow[b]{3}{*}{ Frankreich } & \multirow{3}{*}{$\begin{array}{l}\text { Studiendesigne } \\
\text { Fallkontrollstudie }\end{array}$} & \multirow{3}{*}{$\begin{array}{l}\text { N (TaTME/ } \\
\text { LapTME) } \\
32 / 32\end{array}$} & \multicolumn{5}{|c|}{ Konversionen zur offenen Chirurgie } \\
\hline & & & & \multicolumn{2}{|c|}{ TaTME } & \multicolumn{2}{|c|}{ LapTME } & \multirow{2}{*}{$\begin{array}{l}\boldsymbol{p} \\
1\end{array}$} \\
\hline & & & & $1 / 32$ & $3,1 \%$ & $1 / 32$ & $3,1 \%$ & \\
\hline Fernandez-Hevia 2015 [16] & Spanien & Historische Kontrolle & $37 / 37$ & $0 / 37$ & $0 \%$ & $0 / 37$ & $0 \%$ & 1 \\
\hline Chen 2016 [17] & Taiwan & Fallkontrollstudie & $50 / 100$ & $1 / 50$ & $2 \%$ & $5 / 100$ & $5 \%$ & 0,38 \\
\hline Rasulov 2016 [18] & Russland & Nicht spezifiziert & $22 / 23$ & $0 / 22$ & $0 \%$ & $1 / 23$ & $4 \%$ & 1 \\
\hline LeLong 2017 [19] & Frankreich & Historische Kontrolle & $34 / 38$ & $1 / 34$ & $2,9 \%$ & $9 / 38$ & $23,7 \%$ & 0,015 \\
\hline Perdawood 2018 [20] & Dänemark & $\begin{array}{l}\text { Fallkontrollstudie } \\
\text { (Propensity-Score) }\end{array}$ & $100 / 100$ & $0 / 100$ & $0 \%$ & $11 / 100$ & $11 \%$ & $<0,001$ \\
\hline Persiani 2018 [21] & Italien & $\begin{array}{l}\text { Fallkontrollstudie } \\
\text { (Propensity-Score) }\end{array}$ & $46 / 46$ & $0 / 46$ & $0 \%$ & $9 / 46$ & $19,6 \%$ & 0,002 \\
\hline \multicolumn{3}{|c|}{ Summe aller vergleichenden Studien } & $321 / 376$ & $3 / 321$ & $0,9 \%$ & $36 / 376$ & $9,6 \%$ & 0,001 \\
\hline
\end{tabular}

der COLOR 2-Studie lagen die Raten bei $12 \%$ versus $8 \%$ [1], in der australischen ALaCaRT-Studie bei $8 \%$ versus $13 \%$ [3] und in der amerikanischen ACOSOGZ6051-Studie bei $18 \%$ versus $29 \%$ jeweils in der offenen bzw. laparoskopischen Gruppe $[2,5]$. In der OCUM-Studie war unter den Patienten mit neoadjuvanter Therapie der Anteil nichtkompletter TME-Präparate in durchweg hochspezialisierten Zentren mit $13 \%$ ebenfalls nicht optimal [6].

Auch der Nachweis von Resten des Mesorektums im kleinen Becken in T2gewichteten MRTs bei $36 \%$ der Patienten nach kontinenzerhaltender TME beim Rektumkarzinom aus einem der führenden Zentren Dänemarks deutet darauf hin, dass bei rund einem Drittel der Patienten keine optimale Resektionsqualität vorgelegen hatte [7]. Bemerkenswert ist, dass es sich bei all diesen Studien um prospektive Studien handelt, in denen die Patienten zum weit überwiegenden Teil in Zentren mit besonderer Expertise und lange nach den landesweiten Schulungen der Technik der TME behandelt wurden.

Ein besonders hohes Risiko für suboptimale Resektionen auch nach Konversionen und technisch begründeten Exstirpationen finden sich vor allem bei adipösen Männern mit tiefsitzenden, lokal fortgeschrittenen Rektumkarzinomen. Insbesondere das T-Stadium und damit die Tumorgröße und eine Lokalisation des Tumors bei $7-8 \mathrm{~cm}$ oder weniger sind als Risikofaktoren für eine inkomplette TME gut dokumentiert $[8,9]$. Ein weiterer Risikofaktor ist die vorausgegangene lokale Exzision. Einer aktuellen Metaanalyse zufolge sind in dieser Situation sowohl die Rate inkompletter TMEs als auch die Morbidität des Eingriffs signifikant erhöht [10]. Zudem ist eine signifikante Erhöhung der Exstirpationsrate nach vorausgegangener lokaler Exzision beschrieben [11].

\section{In vielen Studien zur minimal- invasiven TME finden sich hohe Konversionsraten}

In vielen publizierten Studien werden die onkologischen Ergebnisse nur mit hohen Konversionsraten zur offenen Chirurgie bei allein laparoskopischem Vorgehen erreicht. In der COLOR-2-Studie lag die Konversionsrate bei $17 \%$, in der australischen ALaCaRT-Studie bei 9\%, in der amerikanischen ACOSOG-Z6051Studie bei $11 \%$. Auch in der ROLARRStudie, die zum Vergleich von robotischer und nichtrobotischer minimal-invasiver TME mit dem primären Zielkriterium der Konversionsrate initiiert wurde, lag diese bei 8,1 \% (robotisch) bzw. 12,1 \% (laparoskopisch) $(p=0,016)$ [12]. Eindeutig führen Konversionen zu einer Verlängerung der Rekonvaleszenz. Es gibt aber auch eine Reihe von Hinweisen darauf, dass Konversionen bei laparoskopischen Operationen des Rektumkarzinoms mit einer Erhöhung der Anastomoseninsuffizienzrate $[13,14]$ und mit einer Verschlechterung des tumorfreien und Gesamtüberlebens einhergehen [13]. Daher ist $\mathrm{zu}$ betonen, dass sich in den vorliegenden vergleichenden Untersuchungs- serien für die TaTME eine kumulative Konversionsrate von unter $1 \%$ findet, während in den laparoskopischen Armen Konversionsraten von etwa $10 \%$ beschrieben sind (• Tab. 1).

\section{Mögliche Vorteile des transanalen Zugangs bei bestimmten Patientengruppen}

Bereits 2014 wurden im Rahmen einer Konsensuskonferenz zur TaTME folgende lokale anatomische und pathologische Faktoren definiert, die auf mögliche Schwierigkeiten bei der konventionellen TME hindeuteten und die deshalb möglicherweise eine TaTME als bevorzugte Technik erscheinen lassen: männliches Geschlecht, Rekumkarzinom $\leq 12 \mathrm{~cm}$, enges, tiefes Becken, Adipositas, Tumordurchmesser $>4 \mathrm{~cm}$, Fibrose durch vorausgegangene Radiatio, sehr tiefer Tumor mit konsekutiv unsicherem distalen Resektionsrand bei abdominalem Verfahren (insbesondere bei klinisch sehr guter Response) ([22]; - Abb. 1). Im Rahmen einer aktuelleren Konsensuskonferenz wird bezüglich der Patientenselektion festgehalten, dass eine TaTME bei Patienten mit engem Becken, Adipositas und solchen mit voluminösen Tumoren des unteren und mittleren Drittels die technisch erfolgsversprechender durchführbare Methode sein kann [23].

Es bestehen somit Selektionskriterien für Patienten, die wahrscheinlich von dem Verfahren profitieren. Diese sind jedoch weder evidenzbasiert noch all- 
gemein akzeptiert. Auch gibt die Literatur die genannten Selektionskriterien nicht immer richtig wieder. Zwar existieren mehrere Beobachtungsstudien, in denen die Methode nur für tiefsitzende Tumoren eingesetzt wurde [24-26], aber eine strenge Patientenselektion mit z. B. im Median 4 solcher Risikofaktoren (Streubreite 1-6) [27] ist selten exakt dokumentiert und aus den Registerdaten des TaTME-Registers (www.tatme. medicaldata.eu) nicht ohne Weiteres ableitbar [27]. Allerdings ist bei der Verteilung der Tumorhöhen aus diesen $\mathrm{Re}$ gisterdaten $\mathrm{zu}$ berücksichtigen, dass sie zu einem erheblichen Teil die Lernkurve vieler Zentren beinhaltet und explizit die Empfehlung besteht, anfangs nicht mit den Risikopatienten mit besonders tiefen Karzinomen zu beginnen.

\section{Dokumentierte Vorteile des transanalen Zugangs}

Unabhängig von der Entwicklung der minimal-invasiven, videoendoskopisch unterstützten TaTME gibt es in der Literatur eine Reihe gut dokumentierter Hinweise darauf, dass eine Kombination mit einem transanalen Zugang zur Verbesserung der Ergebnisse sinnvoll sein kann. Ein gutes Beispiel dafür ist die Arbeit von Denost et al. [28], die vielfach in Übersichtsarbeiten fälschlich als TaTME-Studie behandelt wird. Hierbei handelt es sich um eine Untersuchung zu einer Operationstechnik, die in der Literatur oft unter dem Begriff „TATA-“ für „transanal-transabdominale“ Rektumresektion beschrieben wird. In der Studie wurde in einem Kollektiv von 100 Patienten mit Karzinomen ausschließlich des unteren Rektumdrittels untersucht, ob die laparoskopische TME durch ein offenes transanales Resezieren des Rektums nach konventionell offener transanaler Präparation der kloakogenen Endstrecke die Ergebnisse der TME im Vergleich zum klassischen Vorgehen mit Stapleranastomose verbessern kann. Tatsächlich fand sich eine signifikant niedrigere Rate von CRM-positiven Präparaten beim kombinierten abdominallaparoskopischen und offen transanalen Vorgehen mit $4 \%$ vs. $18 \%(p=0,025)$. Dieses resultiert im Langzeitverlauf in

coloproctology 2019 · 41:272-279 https://doi.org/10.1007/s00053-019-0362-0

(c) Springer Medizin Verlag GmbH, ein Teil von Springer Nature 2019

A. D. Rink · F. Aigner - M. Biebl · A. Fürst - W. Kneist

\section{Transanale totale mesorektale Exzision - eine sinnvolle Operationstechnik zur individualisierten Behandlung von Patienten mit Rektumkarzinom}

\section{Zusammenfassung}

Hintergrund. Trotz einer erheblichen Verbesserung der Resektionsqualität in der operativen Behandlung des Rektumkarzinoms besteht noch Optimierungspotenzial. Die transanale totale mesorektale Exzision (TaTME) ist ein neues Verfahren, das durch eine Kombination aus abdomineller und minimal-invasiv transanaler Operation diesem Ziel dienen soll.

Ziel. Die Rationale für einen möglichen Nutzen der TaTME zur Verbesserung der Chirurgie des Rektumkarzinoms wird anhand der vorliegenden Evidenz dargestellt.

Ergebnisse. In der Literatur findet sich eine Reihe von Hinweisen auf eine Verbesserung onkologischer Surrogatparameter durch eine kombinierte abdominale und transanale Operation. Einzelne Studien zeigen auch eine Verbesserung dieser Parameter speziell durch die TaTME. Bisher existieren jedoch keine klinischen Langzeitergebnisse, die eine Verbesserung des onkologischen oder funktionellen Outcomes belegen. Überzeugend gezeigt werden konnte jedoch, dass mit der TaTME das Rektumkarzinom minimal-invasiv mit nahezu vollständiger Vermeidung von Konversionen ( $<1 \%)$ erfolgen kann. Die TaTME ist ein eigenständiges Operationsverfahren, welches curricular erlernt werden muss und auch erlernt werden kann. Nach einem entsprechenden Training kann die Methode mit niedriger Morbidität und guten Kurzzeitergebnissen eingeführt werden.

Schlussfolgerungen. Die TaTME ist ein curricular zu erlernendes minimal-invasives Operationsverfahren, welches neben dem Vorteil der nahezu vollständigen Vermeidung von Konversionen für technisch schwer zu operierende Patienten potenzielle Vorteile bietet, die sich bisher aber nur an onkologischen Surrogatparametern, nicht jedoch an klinisch-onkologischen oder funktionellen Daten belegen lassen.

Schlüsselwörter

Rektumresektion · Rektumkarzinom · TAMIS . Transanale Operation $\cdot$ Konversionsrate

\section{Transanal total mesorectal excisiona useful tool for the individualized treatment of patients with rectal cancer}

\section{Abstract}

Background. In spite of significant advances in rectal cancer surgery, there is room and need for further improvement. Transanal total mesorectal excision (TaTME) is a new surgical treatment option that might help improve outcomes by a combined abdominal and transanal approach.

Aim. The rationale and evidence for a potential improvement of rectal cancer treatment by TaTME is presented.

Results. Several studies demonstrate that oncologic surrogate parameters can be improved by a combined abdominal and perineal surgical approach. This is also true specifically for the TaTME technique. However, there are still no clinical outcome data supporting an improvement of both oncologic or functional results. Several comparative trials have shown that conversion to open surgery can almost completely be avoided when patients are treated with TaTME (conversion rate $<1 \%$ ). TaTME needs curricular training in order to avert specific complications. After step-up training, TaTME can be established with good short-term results.

Conclusion. TaTME is a minimally invasive surgical technique for the treatment of rectal cancer that needs curricular training. Aside from nearly complete avoidance of conversion to open surgery, other potential benefits exist that have, however, not been confirmed by clinical oncologic or functional outcome data.

\section{Keywords}

Rectal cancer - Rectal resection - TAMIS .

Transanal surgery $\cdot$ Conversion rate 

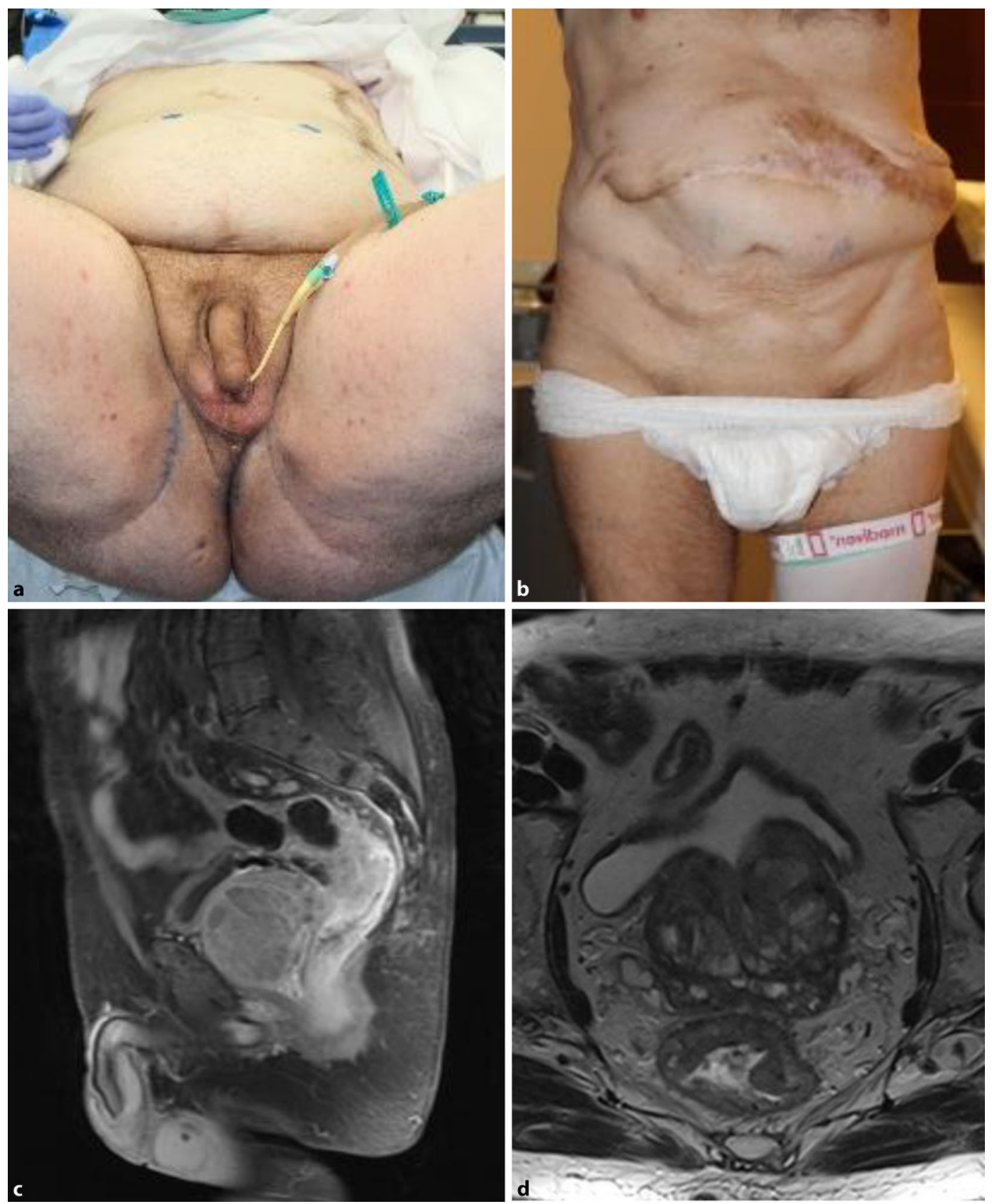

Abb. $1<$ Patienten, die potenziell von der TaTME profitiert haben. a Patient mit morbider Adipositas (BMI $37 \mathrm{~kg} / \mathrm{m}^{2}$ ), b Patient mit supraanalem Rektumkarzinom mit Zustand nach komplikativem Verlauf nach Lebertransplantation und Bauchdeckenersatz durch Bio-Mesh; die laparoskopische Resektion ist durch massive Verwachsungen und die daraus resultierende Unmöglichkeit der Trokarplatzierung in Standardpositionen erheblich erschwert; c, d Mäßig adipöse Patienten mit massiver Prostatahypertrophie

einer niedrigen Lokalrezidivrate von $3 \%$ (gegenüber $5 \% ; p>0,05$ ), ohne dass das funktionelle Ergebnis hierdurch negativ beeinflusst wurde [28, 29]. Zwar ist die Rate von 18\% CRM-positiven Resektaten in der LapTME-Gruppe relativ hoch, allerdings muss berücksichtigt werden, dass es sich um ein Kollektiv von Patienten mit ausschließlich tiefsitzenden Karzinomen handelt. In der OCUM-Studie beispielsweise lag die Rate positiver zirkumferenzieller Resektatränder (CRM) bei Karzinomen des unteren Drittels auch bei knapp über
$10 \%(21 / 205)$, obwohl, ähnlich wie in der französischen Arbeit, fast $80 \%$ der Patienten neoadjuvant radiochemotherapiert wurden [6]. Auch die Arbeit von John und Gerald Marks, den weltweiten Protagonisten des kombinierten offen transanalen und transabdominalen Vorgehens („TATA“) beim Rektumkarzinom, ist keine Arbeit zur TaTME [30]. Sie zeigt aber eindrucksvoll, dass hervorragende Ergebnisse wie eine niedrige Lokalrezidivrate von 7,9\% nach mehr als 5 Jahren Nachbeobachtungszeit bei Patienten mit überwiegend tiefsitzenden
Rektumkarzinomen mit dieser Technik erreicht werden können.

Der Beleg für das Verbesserungspotenzial durch die TaTME findet sich auch in der oben bereits zitierten dänischen Arbeit zur MR-basierten Überprüfung der Vollständigkeit der TME [7]. Während bei $36 \%$ der Patienten mit kontinenzerhaltender TME residuales Mesorektum nachgewiesen wurde, war dies nur bei $13 \%$ der Patienten der Fall, bei denen die Resektion durch eine abdomino-perineale Rektumexstirpation (APR), und damit ebenfalls durch ein kombiniert 
Tab. 2 Kurzzeitergebnisse in vergleichenden Studien zur TaTME vs. LapTME

\begin{tabular}{|c|c|c|c|c|c|c|c|c|c|}
\hline & & $\begin{array}{l}\text { Kontroll- } \\
\text { gruppe }\end{array}$ & & $\begin{array}{l}\text { OP-Zeit } \\
\text { (Minuten) }\end{array}$ & $\begin{array}{l}\text { Hospitali- } \\
\text { sierung } \\
\text { (Tage) }\end{array}$ & $\begin{array}{l}\text { Wiederauf- } \\
\text { nahmen } N / n \\
(\%)\end{array}$ & $\begin{array}{l}\text { Morta- } \\
\text { lität }\end{array}$ & $\begin{array}{l}\text { Morbidität } \\
N / n(\%)\end{array}$ & $\begin{array}{l}\text { Anastomosen- } \\
\text { insuffizienzen } \\
N / n(\%)\end{array}$ \\
\hline \multirow{2}{*}{$\begin{array}{l}\text { Fernandez-Hevia } \\
2015 \text { [16] }\end{array}$} & \multirow[t]{2}{*}{ Spanien } & \multirow[t]{2}{*}{ Historisch } & 37 TaTME & $215^{*}$ & 6,8 & $2 / 37(5,4)$ & 0 & $12 / 37(32)$ & $0 / 37(0)$ \\
\hline & & & 37 LapTME & 252 & 9 & $8 / 37(21,6)$ & 0 & $19 / 37(51)$ & $2 / 37(5,4)$ \\
\hline \multirow{2}{*}{$\begin{array}{l}\text { De'Angelis } 2015 \\
\text { [15] }\end{array}$} & \multirow[t]{2}{*}{ Frankreich } & \multirow[t]{2}{*}{ Matched Pair } & 32 TaTME & $195^{*}$ & 7,8 & $2 / 32(6,2)$ & 0 & $8 / 32(25)$ & $3 / 32(9,4)$ \\
\hline & & & 32 LapTME & 225 & 9,8 & $3 / 32(9,4)$ & 0 & $12 / 32(37,5)$ & $7 / 32(21,9)$ \\
\hline \multirow[t]{2}{*}{ Chen 2016 [17] } & \multirow[t]{2}{*}{ Taiwan } & \multirow[t]{2}{*}{ Matched Pair } & 50 TaTME & 182 & 7,4 & $3 / 50(6)$ & 0 & $10 / 50(20)$ & $3 / 50(6)$ \\
\hline & & & 100 LapTME & 179 & 7,1 & $10 / 100(10)$ & 0 & $17 / 100(17)$ & $4 / 100(4)$ \\
\hline \multirow[t]{2}{*}{ Rasulov 2016 [18] } & \multirow[t]{2}{*}{ Russland } & \multirow[t]{2}{*}{ k. A. } & 22 TaTME & 320 & 8 & k. A. & 0 & $6 / 22(27,3)$ & $0 / 22(0)$ \\
\hline & & & 23 LapTME & 305 & 8 & k. A. & 0 & $6 / 23(26,1)$ & $1 / 23(4,3)$ \\
\hline \multirow[t]{2}{*}{ LeLong 2017 [19] } & \multirow[t]{2}{*}{ Frankreich } & \multirow[t]{2}{*}{ Historisch } & 34 TaTME & $532^{\mathrm{a}}$ & $8 *$ & $0 / 34(0)^{*}$ & 0 & $11 / 34(32)$ & $2 / 34(5,9)$ \\
\hline & & & 38 LapTME & 576 & 9 & $6 / 38(15,8)$ & 0 & $14 / 38(35)$ & $6 / 38(15,8)$ \\
\hline \multirow{3}{*}{$\begin{array}{l}\text { Perdawood } 2018 \\
\text { [20] }\end{array}$} & \multirow[t]{3}{*}{ Dänemark } & \multirow{3}{*}{$\begin{array}{l}\text { Propensity- } \\
\text { matched pair }\end{array}$} & 100 TaTME & $285^{* * *}$ & $8,6^{* *}$ & $14 / 100(14)^{*}$ & 0 & $12 / 100(12)^{* b}$ & $6 / 63(9,5)$ \\
\hline & & & 100 LapTME & 334 & 14,2 & $28 / 100(28)$ & 0 & $24 / 100(24)$ & $11 / 66(16,7)$ \\
\hline & & & 100 opTME & 325 & 15,5 & $20 / 100(20)$ & 0 & $30 / 100(30)$ & $17 / 66(25,8)$ \\
\hline \multirow[t]{2}{*}{ Persiani 2018 [21] } & \multirow[t]{2}{*}{ Italien } & \multirow{4}{*}{$\begin{array}{l}\text { Propensity- } \\
\text { matched pair }\end{array}$} & 46 TaTME & 276 & $5^{* *}$ & k. A. & 0 & $11 / 46(23,9)$ & $3 / 46(7,5)$ \\
\hline & & & 46 LapTME & 272 & 7 & k. A. & 0 & $10 / 46(21,7)$ & $2 / 46(4,5)$ \\
\hline \multirow{2}{*}{$\begin{array}{l}\text { Summe aller } \\
\text { vergleichenden } \\
\text { Studien }\end{array}$} & & & 321 TaTME & $279^{(\text {n.b. })}$ & $7,5^{(\text {n.b. })}$ & $21 / 253(8,3)^{* * *}$ & 0 & $70 / 321(21,8)$ & $17 / 284(6)$ \\
\hline & & & 376 LapTME & 291 & 9,6 & $55 / 307(17,9)$ & 0 & $\begin{array}{l}102 / 376 \\
(27,1)\end{array}$ & $33 / 342(9,6)$ \\
\hline \multicolumn{10}{|c|}{ 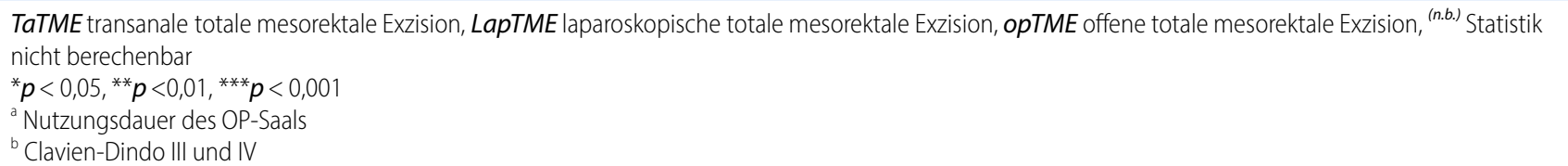 } \\
\hline
\end{tabular}

abdominal-perineales Verfahren erfolgte [7]. In einer aktuell publizierten holländischen Vergleichsstudie konnte nun gezeigt werden, dass residuales Mesorektum in MRTs bei $46,9 \%$ der Patienten nach laparoskopischer TME, aber nur in $3,1 \%$ nach TaTME nachgewiesen wurde $(p<0,0001)$ [31]. Letztlich deuten aktuelle Studien auch auf deutliche Vorteile der transanalen Technik bei der SalvageTME nach vorausgegangener lokaler Exzision (z. B. transanale endoskopische Mikrochirurgie [TEM]) hin [32, 33].

Weitere potenzielle Vorteile der TaTME sind in - Tab. 2 dargestellt. Neben einer Reduktion der Operationszeit, die jedoch nur durch Durchführung der Operation mit zwei Teams erreicht wird, zeigen sich in einigen Studien signifikante Vorteile bezüglich der Morbidität, der postoperativen Verweildauer und der Wiederaufnahmerate, wobei Letztere auch in der kumulativen Statistik aller vergleichenden Studien signifikant ist.

\section{Onkologische Ergebnisse}

Ein onkologischer Vorteil der TaTME ist bisher nicht belegt. Immerhin sind aber die bisher vorliegenden onkologischen Surrogatparameter vielversprechend. In einer aktuellen Metaanalyse wird für die TaTME im Vergleich zur LapTME ein signifikant weiterer aboraler Sicherheitsabstand (DRM: distal resection margin) beschrieben. Dieser Aspekt ist differenziert aus diagnostischer, operationstechnischer, onkologischer und funktioneller Sicht kritisch zu betrachten. Bereits bei der Operationsplanung sind eine Angabe zum intendierten DRM (inklusive akzeptiertes Minimum \& Maximum) und weiter die separate Angabe des DRM für sphinkter-bzw. kontinenzerhaltende und nicht-kontinenzerhaltende TMEs erforderlich [34]. Zukünftig könnte der Anteil der Patienten, bei denen der intendierte DRM erreicht wurde, als Surrogatparameter dienen, nicht zuletzt um Operationsresultate besser vergleichen zu können [34]. Funktionelle Chancen und Risi- ken müssen vor dem Hintergrund des individuellen Patientenwunsches und der teilweise sehr guten Tumorregressionen nach neoadjuvanter Therapie (klinisch komplettes Ansprechen) absehbar häufiger in Tumorboards und im Rahmen der Gewährung von Zweitmeinungsverfahren erläutert werden. Neue Erkenntnisse zur wirksamen Therapie bei postoperativen funktionellen Einschränkungen z. B. beim LARS(, lower anterior resection syndrom") - können dabei zunehmend eingebracht werden [35].

Über den DRM hinaus wird auf signifikant weitere zirkumferenzielle Resektatränder (CRM) und eine signifikant niedrigere Quote an positiven CRM hingewiesen [36]. Die noch wenigen onkologischen Verlaufsdaten sind zumindest vielversprechend. Beispielsweise fanden Tuech und Mitarbeiter in einer konsekutiven Serie von 54 Patienten mit ausschließlich kontinenzerhaltend resezierten Karzinomen des unteren Rektumdrittels bei einer mittleren Nachbeobachtungszeit von 29 Mona- 
ten nur 1,8\% Lokalrezidive und 3,6\% Fernmetastasen [25]. Derzeit werden in zwei europäischen Studien Patienten zwischen TaTME und LapTME randomisiert (COLOR-3 und GRECCAR-11 [37, 38]), um der fehlenden Evidenz zu begegnen. Die TaTME wird die laparoskopische TME ebenso wenig wie die robotische TME ersetzen. In den angesprochenen randomisierten Studien wird auf Subgruppenanalysen in den Risikokollektiven geschaut werden müssen. Die Vorteile der TaTME werden vermutlich erst dann erkennbar werden.

\section{Funktionalität}

Bisher können keine klaren Vorteile für die TaTME bezüglich der Funktionalität aus den bisherigen Daten abgeleitet werden. Einzelnen Berichten über eine bessere urogenitale Funktion nach TaTME im Vergleich zur LapTME stehen Hinweise auf eine möglicherweise sogar schlechtere anorektale Funktion gegenüber [39]. $\mathrm{Zu}$ berücksichtigen ist dabei, dass bei den vergleichenden Studien unterschiedliche Nachuntersuchungsintervalle in der TaTME und der Vergleichsgrupppe vorliegen. So ist in der Arbeit aus Dänemark der mediane Nachuntersuchungszeitraum in der TaTMEGruppe mit 23 Monaten erheblich kürzer als der in der LapTME-Gruppe mit 75 Monaten $(p<0,001)$ [39]. Das gleich gilt für die holländische Arbeit, in der der Unterschied mit 20 vs. 59 Monaten ebenfalls deutlich war $(p<0,001)$ [40], ebenso wie für die französische Arbeit mit 32 vs. 53 Monaten $(p<0,01)$ [29]. Dies ist insofern bedeutsam, als die durch die autonomen Beckennerven vermittelten Funktionen sich mit fortschreitendem Abstand zur OP zumindest teilweise verbessern können [41].

Zudem fallen im Langzeitverlauf oft Patienten aus dem Kollektiv, bei denen wegen schlechterer Funktion ein definitives Stoma angelegt wurde. Da keine kontrollierten Studien mit primären funktionellen Endpunkten vorliegen und die vergleichenden Arbeiten durch die unterschiedlichen Nachuntersuchungszeiträume praktisch nicht zu bewerten sind, bleibt leider die Frage nach den diesbezüglichen Vor- oder Nachteilen der TaTME unbeantwortet.

\section{TaTME - neue Technik, neue Risiken?}

Bei einem neuen operativen Verfahren muss die Frage nach methodenspezifischen neuen Risiken gestellt werden. Hier ist am intensivsten die Urethraverletzung diskutiert, wobei zu hinterfragen ist, ob es sich hierbei tatsächlich um eine TaTMEspezifische Komplikation handelt. Das Risiko für eine Urethraverletzung muss nach den publizierten Registerdaten mit 1,1 \% für die männlichen Patienten angegeben werden [27]. Berücksichtigt werden sollte hier aber, dass in dem Register in großem Umfang Patienten während der Lernkurve der Operateure (zum großen Teil frühe Anwender) eingeschlossen wurden. Noch wichtiger ist aber, dass es auch bei anderen Operationstechniken für das Rektumkarzinom zu Urethraverletzungen kommen kann, wie zum Beispiel bei der Rektumexstirpation. In der Literatur findet sich eine Arbeit, die für die offene APR ein Risiko von knapp $2 \%$ angibt [42]. Auch bei der anterioren Resektion können urethrale Verletzungen auftreten. Dies zeigt beispielsweise die Arbeit von Ng und Mitarbeitern [43]. Es wird über zwei Urethraverletzungen bei 579 laparoskopischen kolorektalen Resektionen berichtet. Schließt man jedoch die in dieser Studie eingeschlossenen Karzinome des oberen Rektumdrittels und Sigmas aus, so ergibt sich für die etwa 132 männlichen Patienten mit einem Tumor bis $12 \mathrm{~cm}$ ein Risiko von 1,5\%, sodass diese Arbeit die Urethraläsion als nicht ausschließlich TaTMEassoziierte Komplikation belegt.

Nichtsdestoweniger besteht bei tiefen Karzinomen sicher ein Risiko der Urethraverletzung, und entsprechende Vermeidungsstrategien werden in den nationalen und internationalen TaTME-Kursen auch intensiv geschult $[44,45]$.

Die rezent berichteten erhöhten multifokalen Rezidivraten nach TaTME an einigen großteils Low-Volume-Zentren (Norwegen, Holland) zeugen von der eingehaltenen Transparenz der internationalen Community und einem ernstzunehmenden onkologischen Risiko bei der initialen Einführung bzw. in der Lernkurve der TaTME (Sietses C. Persönliche Mitteilung: Innovations in Colorectal Surgery; Amsterdam 29.03.2019).

Insgesamt bleibt festzuhalten, dass die TaTME eine anspruchsvolle Technik ist, die curricular erlernt werden muss. Unter deutscher Beteiligung wurden bereits entsprechende Ausbildungscurricula publiziert [46]. In Anlehnung an diese internationalen Empfehlungen haben wir auch in Deutschland ein entsprechendes Curriculum erstellt, welches folgende Teilschritte umfasst: 1) Selbststudium über Videos, Kongresse, Publikationen und Hospitationen, 2) einen Trainingskurs mit Operationen am Körperspender und 3) ein Proctoring-Programm, welches eine Begleitung der ersten Operationen des auszubildenden Chirurgen durch einen TaTME-erfahrenen Operateur vorsieht. So wird in Deutschland seit drei Jahren eine Reihe von Hospitationskursen und Körperspenderkursen angeboten, und auch ein durch Studien mittlerweile belegtes Proctoring-Programm [47] wird absehbar in Zusammenarbeit mit der Deutschen Gesellschaft für Allgemein- und Viszeralchirugie angeboten werden können. Im Rahmen der Kurse wird mittlerweile "step by step“ TaTME geschult. Im Rahmen einer internationalen Konsensuskonferenz in St. Gallen wurden die Standards erarbeitet, sie sind mittlerweile publiziert und werden, wie man an den nahezu europaweit inhaltsgleichen Kursen erkennen kann, auch in weitem Umfang so umgesetzt [23]. Nach Durchlaufen eines entsprechenden Ausbildungscurriculums ist die sichere Einführung der Methode an High-Volume Zentren möglich [47, 48].

\section{Fazit}
- Der Bedarf für eine weitere Optimie- rung der Operationstechnik über die konventionelle und laparoskopische TME hinaus wird durch die große Zahl suboptimal resezierter Patienten in großen Studien belegt.
- Die TaTME ist ein mittlerweile hoch standardisiertes Operationsverfah- ren, welches dem in der Rektumchir- urgie erfahrenen Viszeralchirurgen potenzielle Vorteile bietet, um bei


absehbar technisch schwer zu operierenden Patienten eine optimale onkologische Resektion und, soweit funktionell sinnvoll, auch den Erhalt der Darmkontinuität zu ermöglichen. Dies kann unter nahezu vollständiger Vermeidung von Konversionen zur offenen Chirurgie erfolgen.

- Die Technik der TaTME muss weiter intensiv studiert werden. Sie kann erst nach einem entsprechend durchlaufenen Ausbildungscurriculum sicher eingeführt und praktiziert werden. Die TaTME ist keine Operationsmethode für jeden Chirurgen und auch nicht für jeden Patienten mit Indikation zur TME bei Rektumkarzinom. Nach einer entsprechenden Schulung ist sie aber zumindest bei solchen Resektionen sinnvoll und vertretbar anwendbar, die ohnehin zu einer supraanalen oder intersphinktären Anastomose führen und daher von spezialisierten Chirurgen durchgeführt werden sollten.

\section{Korrespondenzadresse}

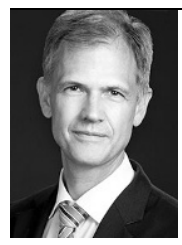

PD Dr. A. D. Rink

Klinik für Allgemein-, Viszeral- und Thoraxchirurgie, Klinikum Leverkusen Am Gesundheitspark 11, 51375 Leverkusen, Deutschland andreas.rink@klinikum-lev.de

\section{Einhaltung ethischer Richtlinien}

Interessenkonflikt. A.D. Rink, F. Aigner, M. Biebl, A. Fürst und W. Kneist geben an, dass kein Interessenkonflikt besteht.

Für diesen Beitrag wurden von den Autoren keine Studien an Menschen oder Tieren durchgeführt. Für die aufgeführten Studien gelten die jeweils dort angegebenen ethischen Richtlinien.

\section{Literatur}

1. van der Pas MH, Haglind E, Cuesta MA, Furst A, Lacy AM, Hop WC et al (2013) Laparoscopic versus open surgery for rectal cancer (COLOR II): short-term outcomes of a randomised, phase 3 trial. Lancet Oncol 14(3):210-218

2. Fleshman J, Branda M, Sargent DJ, Boller AM, George V, Abbas M et al (2015) Effect of Laparoscopic-assisted resection vs open resection of stage II or III rectal cancer on pathologic outcomes: the ACOSOG Z6051 randomized clinical trial. JAMA 314(13):1346-1355

3. Stevenson AR, Solomon MJ, Lumley JW, Hewett P, Clouston AD, Gebski VJ et al (2015) Effect of Laparoscopic-assisted resection vs open resection on pathological outcomes in rectal cancer: the AlacaRT randomized clinical trial. JAMA 314(13):1356-1363

4. Nagtegaal ID, van de Velde CJ, van der Worp E, Kapiteijn E, Quirke P, van Krieken JH et al (2002) Macroscopic evaluation of rectal cancer resection specimen: clinical significance of the pathologist in quality control. JClin Oncol 20(7):1729-1734

5. Spinelli ADHA, Panis $Y$, Bemelman WA, Jayne DG, Fürst A (2017) Critical appraisal of two randomized clinical trials on pathologic outcomes - Laparoscopic vs. open resection for rectal cancer. Coloproctology 39(4):277

6. Kreis ME, Ruppert R, Ptok H, Strassburg J, Brosi P Lewin A et al (2016) Use of preoperative magnetic resonance imaging to select patients with rectal cancer for neoadjuvant chemoradiation-interim analysis of the German OCUM trial (NCT01325649). JGastrointest Surg 20(1):25-32 (discussion-3)

7. Bondeven $\mathrm{P}$, Hagemann-Madsen $\mathrm{RH}$, Laurberg S, Pedersen BG (2013) Extent and completeness of mesorectal excision evaluated by postoperative magnetic resonance imaging. $\mathrm{Br} J$ Surg 100(10):1357-1367

8. Leonard D, Penninckx F, Fieuws S, Jouret-Mourin A, SempouxC, Jehaes Cetal (2010) Factors predicting the quality of total mesorectal excision for rectal cancer. Ann Surg 252(6):982-988

9. Garlipp B, Ptok H, Schmidt U, Stubs P, Scheidbach $\mathrm{H}$, Meyer $\mathrm{F}$ et al (2012) Factors influencing the quality of total mesorectal excision. Br J Surg 99(5):714-720

10. Eid Y, Alves A, Lubrano J, Menahem B (2018) Does previous transanal excision for early rectal cancer impair surgical outcomes and pathologic findings of completion total mesorectal excision? Results of a systematic review of the literature. J Visc Surg 155(6):445-452

11. Morino M, Allaix ME, Arolfo S, Arezzo A (2013) Previous transanal endoscopic microsurgery for rectal cancer represents a risk factor for an increased abdominoperineal resection rate. Surg Endosc 27(9):3315-3321

12. Jayne D, Pigazzi A, Marshall H, Croft J, Corrigan N, Copeland J et al (2017) Effect of roboticassisted vs conventional Laparoscopic surgery on risk of conversion to open laparotomy among patients undergoing resection for rectal cancer: the ROLARR randomized clinical trial. JAMA 318(16):1569-1580

13. Furnee EJB, Aukema TS, Oosterling SJ, Borstlap WAA, Bemelman WA, Tanis PJ et al (2018) Influence of conversion and anastomotic leakage on survival in rectal cancer surgery; retrospective crosssectional study. J Gastrointest Surg https://doi. org/10.1007/s11605-018-3931-6

14. Liu XH, Wu XR, Zhou C, Zheng XB, Ke J, Liu HS et al (2018) Conversion is a risk factor for postoperative anastomotic leak in rectal cancer patients-A retrospective cohort study. Int J Surg 53:298-303

15. de'Angelis N, Portigliotti L, Azoulay D, Brunetti $F$ (2015) Transanal total mesorectal excision for rectal cancer: a single center experience and systematic review of the literature. Langenbecks Arch Surg 400(8):945-959

16. Fernandez-Hevia M, Delgado S, Castells A, Tasende M, Momblan D, Diaz del Gobbo $G$ et al (2015) Transanal total mesorectal excision in rectal cancer: short-term outcomes in comparison with laparoscopic surgery. Ann Surg 261(2):221-227

17. Chen CC, Lai YL, Jiang JK, Chu CH, Huang IP, Chen WS et al (2016) Transanal total mesorectal excision versus laparoscopic surgery for rectal cancer receiving neoadjuvant chemoradiation: a matched case-control study. Ann Surg Oncol 23(4):1169-1176

18. Rasulov AO, Mamedli ZZ, Gordeyev SS, Kozlov NA, Dzhumabaev HE (2016) Short-term outcomes after transanal and laparoscopic total mesorectal excision for rectal cancer. Tech Coloproctol 20(4):227-234

19. Lelong $B$, Meillat $H$, Zemmour C, Poizat $F$, Ewald J, Mege D et al (2017) Short- and midterm outcomes after endoscopic transanal or laparoscopic transabdominal total mesorectal excision for low rectal cancer: a single institutional case-control study. J Am Coll Surg 224(5):917-925

20. Perdawood SK, Thinggaard BS, Bjoern MX (2018) Effect of transanal total mesorectal excision for rectal cancer: comparison of short-term outcomes with laparoscopic and open surgeries. Surg Endosc 32(5):2312-2321

21. Persiani R, Biondi A, Pennestri F, Fico V, De Simone V, Tirelli F et al (2018) Transanal total mesorectal excision vs laparoscopic total mesorectal excision in the treatment of low and middle rectal cancer: a propensity score matching analysis. Dis Colon Rectum 61(7):809-816

22. Motson RW, Whiteford MH, Hompes R, Albert M, Miles WF, Expert G (2016) Current status of transanal total mesorectal excision (TaTME) following the Second International Consensus Conference. Colorectal Dis 18(1):13-18

23. Adamina M, Buchs NC, Penna M, Hompes $R$ (2018) St.Gallen Colorectal Consensus Expert G. St.Gallen consensus on safe implementation of transanal total mesorectal excision. Surg Endosc 32(3):1091-1103

24. RinkAD, KauffDW, Paschold M, Vestweber KH, Lang H, Kneist W (2016) Hybrid TAMIS total mesorectal excision. A new perspective in treatment of distal rectal cancer-Technique and results. Chirurg 87(3):225-232

25. Tuech JJ, Karoui M, Lelong B, De Chaisemartin C, Bridoux V, Manceau G et al (2015) A step toward NOTES total mesorectal excision for rectal cancer: endoscopic transanal proctectomy. Ann Surg 261(2):228-233

26. Muratore A, Mellano A, Marsanic P, De Simone M (2015) Transanal total mesorectal excision (taTME) for cancer located in the lower rectum: short- and mid-term results. Eur J Surg Oncol 41(4):478-483

27. Kneist W, Hanke L, Kauff DW, Lang H (2016) Surgeons' assessment of internal anal sphincter nerve supply during TaTME-inbetween expectations and reality. Minim Invasive Ther Allied Technol 25(5):241-246

28. Denost Q, Loughlin P, Chevalier R, Celerier B, Didailler R, Rullier E (2018) Transanal versus abdominal low rectal dissection for rectal cancer: long-term results of the Bordeaux' randomized trial. Surg Endosc 32(3):1486-1494

29. Pontallier A, Denost Q, Van Geluwe B, Adam JP, Celerier B, Rullier E (2016) Potential sexual function improvement by using transanal mesorectal approach for laparoscopic low rectal cancer excision. Surg Endosc 30(11):4924-4933

30. Marks JH, MyersEA,ZegerEL, Denittis AS, Gummadi M, Marks GJ (2017) Long-term outcomes by a transanal approach to total mesorectal excision for rectal cancer. Surg Endosc 31(12):5248-5257 
31. Veltcamp Helbach $M$, Koedam TWA, Knol JJ, Diederik A, Spaargaren GJ, Bonjer HJ et al (2019) Residual mesorectum on postoperative magnetic resonance imaging following transanal total mesorectal excision (TaTME) and laparoscopic total mesorectal excision (LapTME) in rectal cancer. SurgEndosc 33(1):94-102

32. Letarte F, Raval M, Karimuddin A, Phang PT, Brown CJ (2018) Salvage TME following TEM: a possible indication for TaTME. Tech Coloproctol 22(5):355-361

33. Koedam TWA, Veltcamp Helbach M, Penna M, Wijsmuller A, Doornebosch P, van Westreenen $\mathrm{HL}$ et al (2019) Short-term outcomes of transanal completion total mesorectal excision (cTaTME) for rectal cancer: a case-matched analysis. Surg Endosc 33(1):103-109

34. Rink AD, Kneist W (2019) How to report on distal resection margins in trials on rectal cancer surgery. Dis Colon Rectum 62(1):e1-e2

35. Rosen HR, Kneist W, Fürst A, Krämer G, Hebenstreit J, Schiemer JF (2019) Randomized clinical trial of prophylactic transanal irrigation versus supportive therapy to prevent symptoms of low anterior resection syndrome after rectal resection. Br J Surg. https://doi.org/10.1002/bjs5.50160

36. Jiang HP, Li YS, Wang B, Wang C, Liu F, Shen ZL et al (2018) Pathological outcomes of transanal versus laparoscopic total mesorectal excision for rectal cancer: a systematic review with meta-analysis. Surg Endosc 32(6):2632-2642

37. Lelong $B$, de Chaisemartin C, Meillat $H$, Cournier S, Boher JM, Genre D et al (2017) A multicentre randomised controlled trial to evaluate the efficacy, morbidity and functional outcome of endoscopic transanal proctectomy versus laparoscopic proctectomy for low-lying rectal cancer (ETAP-GRECCAR 11 TRIAL): rationale and design. BMCCancer 17(1):253

38. Deijen $C L$, Velthuis $S$, Tsai A, Mavroveli S, de Lange-de Klerk ES, Sietses C et al (2016) COLOR III: a multicentre randomised clinical trial comparing transanal TME versus laparoscopic TME for mid and low rectal cancer. Surg Endosc 30(8):3210-3215

39. Bjoern MX, Nielsen S, Perdawood SK (2019) Quality of life after surgery for rectal cancer: a comparison of functional outcomes after transanal and laparoscopic approaches. J Gastrointest Surg https://doi.org/10.1007/s11605-018-4057-6

40. Veltcamp Helbach M, Koedam TWA, Knol JJ, Velthuis S, Bonjer HJ, Tuynman JB et al (2019) Quality of life after rectal cancer surgery: differences between laparoscopic and transanal total mesorectal excision. Surg Endosc 33(1):79-87

41. Del Rio C, Sanchez-Santos R, Oreja V, De Oca J, Biondo S, Pares D et al (2004) Long-term urinary dysfunction after rectal cancer surgery. Colorectal Dis 6(3):198-202

42. Andersson A, Bergdahl L (1976) Urologic complications following abdominoperineal resection of the rectum. Arch Surg 111(9):969-971

43. Ng KHND, Cheung HY, Wong JC, Yau KK, Chung CC, Li MK (2009) Laparoscopic resection for rectal cancers: lessons learned from 579 cases. Ann Surg 249(1):82-86
44. Aigner $F$, Biebl $M$, Furst $A$, Jons $T$, Pratschke J, Kneist W (2017) Training course transanal total mesorectal excision (TaTME) : concept and establishment of a training course for safe application. Chirurg 88(2):147-154

45. Kneist W, Aigner F, Fürst A, Wedel T (2017) Urethral injury in body donor TaTME training. Coloproctology 39(3):179-183

46. Francis N, Penna M, Mackenzie H, Carter F, Hompes $\mathrm{R}$, International Ta TMEECG (2017) Consensus on structured training curriculum for transanal total mesorectal excision (TaTME). Surg Endosc 31(7):2711-2719

47. Abbott SC, Stevenson ARL, Bell SW, Clark D, Merrie A, Hayes J et al (2018) An assessment of an Australasian pathway for the introduction of transanal total mesorectal excision (taTME). Colorectal Dis 20(1):01-06

48. Veltcamp Helbach M, van Oostendorp SE, Koedam TWA, Knol JJ, Stockmann H, Oosterling SJ et al (2019) Structured training pathway and proctoring; multicenter results of the implementation of transanal total mesorectal excision (TaTME) in the Netherlands. Surg Endosc. https://doi.org/10. 1007/s00464-019-06750-w

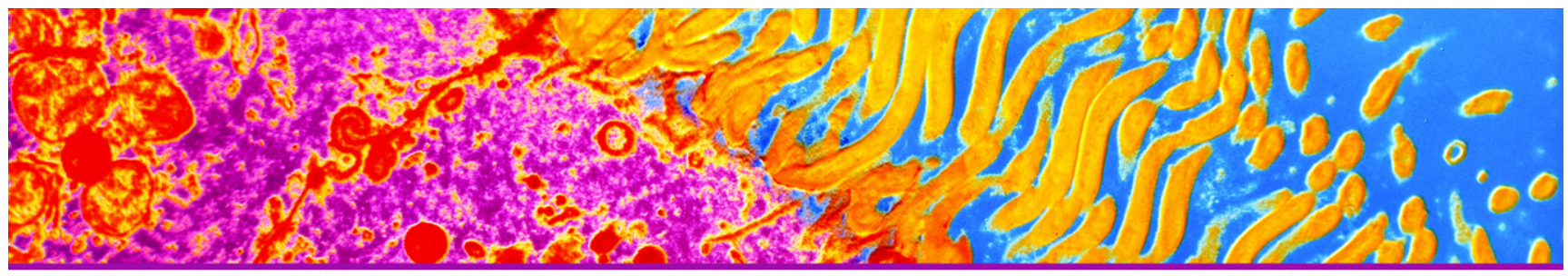

\section{Der neue Kurs zu Syphilis}

Alles Wichtige kompakt und aktuell auf den Punkt gebracht!

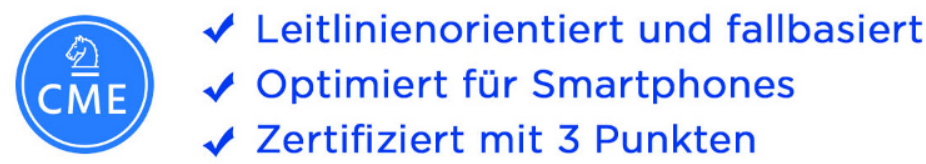

Diesen CME-Kurs finden Sie auf

\section{» DGIM-eAkademie.de}

Das Fortbildungs-Portal der DGIM:
Kostenfrei für alle Mitglieder und e.Med-Abonennten 